\title{
Exploring Early Christian Identity
}

\section{Ed. by Bengt Holmberg}

[Frühchristliche Identität.]

Veröffentlicht auf Englisch.

Die Autoren der Beiträge des vorliegenden Bandes untersuchen die frühchristliche Identität als einen Schlüssel zum

Exploring Early Christian Identity

Edited by BENGT HOLMBERG

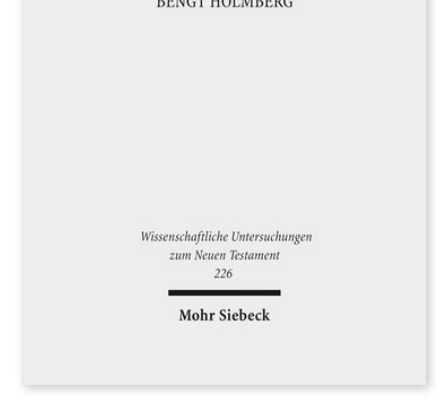

2008. VIII, 205 Seiten. WUNT I 226

ISBN 978-3-16-151515-6

DOI 10.1628/978-3-16-151515-6

eBook PDF $79,00 €$

ISBN 978-3-16-149674-5

Leinen $79,00 €$
Verständnis der Entwicklung des frühen Christentums. Dieses wandelte sich von einer inneriüdischen Erneuerungsbewegung zu einer neuen, individuellen Religion mit größtenteils nichtjüdischen Mitgliedern und einigen bedeutenden Unterschieden zu ihrer jüdischen Form. Identität besteht nicht nur aus einer Ansammlung von Glaubensmerkmalen, sondern stellt vielmehr eine Beteiligung an den Kulten, Riten, der ethischen Lehre und an Verhaltensnormen dar, die in sozialen Beziehungen und Institutionen Gestalt annehmen. Dies ist ein dynamischer Rückkoppelungsprozess, der Konflikte und Schwierigkeiten beinhaltet, und der sowohl innerhalb der Gruppe besteht als auch durch die umgebende Gesellschaft und Kultur hervorgerufen wird. Die Beiträger des Bandes behandeln unterschiedliche Aspekte dieses Phänomens. Sie analysieren das soziale Gedächtnis, die Klassifizierung, die soziale Wahrnehmung, Führungsideale, das moralische Lehren wie auch Geschlechteraspekte.

\section{Inhaltsübersicht}

Bengt Holmberg: Understanding the First Hundred Years of Christian Identity - Samuel Byrskog: Memory and Identity in the Gospels: A New Perspective - Anders Runesson: The Invention of Christian Identity: Paul, Ignatius, and Theodosius- Rikard Roitto: Behaving like a Christ-Believer. A Cognitive Perspective on Identity and Behavior Norms in the Early Christ-Movement Mikael Tellbe: The Prototypical Christ-Believer. Early Christian Identity Formation in Ephesus - Runar Thorsteinsson: The Role of Morality in the Rise of Roman Christianity - Fredrik Ivarsson: Christian Identity as True Masculinity - Bengt Holmberg: Early Christian identity - some conclusions

Bengt Holmberg is professor emeritus at Lund University, Sweden.

Jetzt bestellen:

https://mohrsiebeck.com/buch/exploring-early-christian-identity-9783161515156?no_cache=1

order@mohrsiebeck.com

Telefon: $+49(0) 7071-923-17$

Telefax: +49 (0)7071-51104 